\title{
Group-Wise Consistent Parcellation of Gyri via Adaptive Multi-view Spectral Clustering of Fiber Shapes
}

\author{
Hanbo Chen ${ }^{1}$, Xiao $\mathrm{Cai}^{2}$, Dajiang Zhu ${ }^{1}$, Feiping $\mathrm{Nie}^{2}$, \\ Tianming Liu ${ }^{1}$, and Heng Huang ${ }^{2}$ \\ ${ }^{1}$ Department of Computer Science and Bioimaging Research Center, \\ The University of Georgia, Athens, GA, USA \\ ${ }^{2}$ Department of Computer Science and Engineering, \\ University of Texas at Arlington, Arlington, Texas, United States
}

\begin{abstract}
In-vivo parcellation of the cerebral cortex via non-invasive neuroimaging data has been in active research for years. A variety of modeldriven and/or data-driven computational approaches have been proposed to parcellate the cortex. However, two fundamental common issues in these parcellation methodologies are the features or attributes used to define boundaries between cortical regions and the establishment of correspondences of the parcellated regions across different brains. This paper uses a novel DTIderived fiber shape feature for the parcellation of cortical gyrus into finegranularity segments. The gyral parcellation is formulated and solved as a surface vertex clustering problem, in which fiber shape feature similarity is used to define the distances between vertices. Then, we designed and applied a novel multi-view spectral clustering algorithm to group the vertices into groupwise consistent gyral segments across different brains. The experimental results showed that the precentral and postcentral gyrus, as two test-beds, can be consistently parcellated into 10 segments on both hemispheres across different subjects. Evaluation studies using benchmark task-based fMRI and cortical landmarks demonstrated the effectiveness and validity of the proposed methods.
\end{abstract}

Keywords: cortical parcellation, multi-view clustering, fiber shape, gyri.

\section{Introduction}

In-vivo parcellation of the human cerebral cortex based on neuroimaging data, e.g., MRI/DTI/fMRI, has been extensively studied because of its significant importance in basic and clinical neurosciences. In general, cortical parcellation result can be used for the definition of anatomically/connectionally/functionally meaningful structures and for measurement of their biological properties. Current approaches to cortical parcellation can be broadly categorized into two classes: model-driven and datadriven methods. In model-driven methods, atlas-based warping methods [1]-[2] are widely used to parcellate a subject's cortex by transforming an expert-labeled atlas to the subject's space. In data-driven methods, geometrical, morphological, connectional or functional features can be used to guide the parcellation procedure, e.g., in [3], a sulcal parcellation algorithm was developed based on the cortical folding patterns. 
In this paper, we propose a novel approach for fine-granularity cortical gyrus parcellation via group-wise multi-view spectral clustering of fiber shape patterns. The basic idea is that each cortical gyral region is represented by its DTI-derived fiber connection shape patterns, which is able to capture the global structural connectivity pattern and thus is predictive of brain function. Then, the representative nodes of each gyrus from different brains are roughly aligned and each subject represents a view. A novel multi-view spectral clustering algorithm is designed to cluster these gryal nodes into group-wise consistent segments with correspondences across individuals. Here, the similarity matrix of the gyral nodes in each subject is modeled and represented as a separate view in the context of multi-view clustering problems. The major methodological contribution of this work is that the proposed method achieved meaningful parcellation of the gyrus and the establishment of correspondences of the parcellated segments across individuals simultaneously. The neuroscience insight gained from this work is that the seemingly very variable cortical gyrus and its fiber connections can be consistently parcellated into meaningful and common segments. In the future, the derived consistent gyral segments can be used as common ROIs (regions of interests) for a variety of neuroscience applications, such as mapping of structural and functional connectivities in healthy brains and diseased ones.

\section{Methods}

The computational pipeline is summarized in Fig. 1. The major novel steps are in Figs. 1c and 1d. In Fig. 1c, the gyrus is represented by discrete nodes on its extracted crest line and each gyral node is described by rich and functionally meaningful DTIderived fiber connection patterns. In Fig. 1d, the gyral nodes from multiple brains are clustered into group-wise consistent segments by a novel multi-view spectral clustering algorithm. Finally, these DTI-derived consistent segments are validated by benchmark cortical landmarks and task-based fMRI activations on the gyri.

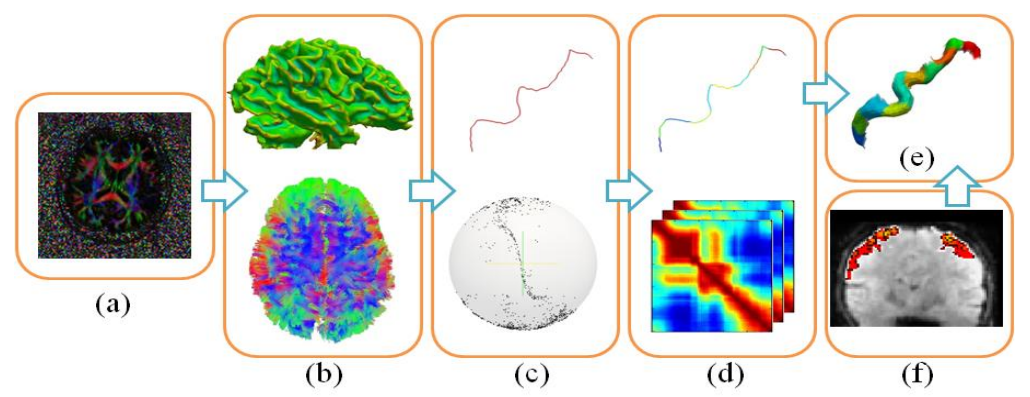

Fig. 1. The computational pipeline of group-wise multi-view clustering of fiber shape patterns for gyral parcellation. (a) DTI raw data. (b) Pre-processing including tissue segmentation, surface reconstruction, and fiber tracking. (c) Extraction of gyral crest lines and corresponding fiber shape features. (d) Group-wise clustering based on multi-view spectral clustering. (e) Gyral parcellation result. (f) Functional activation map for validation. 


\subsection{Data Acquisition and Preprocessing}

Two separate datasets are used for this study. In the first dataset, 6 healthy volunteers were scanned with both DTI and task-based fMRI. The parameters of DTI is as follows: $128 \times 128$ matrix, $2 \mathrm{~mm}$ slice thickness, $256 \mathrm{~mm}$ FOV, 60 slices, TR=15s, ASSET $=2$, 30 DWI gradient directions, 3 B0 images, b-value $=1000$. For fMRI scans, the motor tasks were designed using similar paradigms in [4], in which elbow, lip and ankle movements were performed respectively. The parameters are as follows: $2 \mathrm{~mm}$ isotropic images at TR/TE $=3000 / 25 \mathrm{~ms}$, $\mathrm{FOV}=256 \mathrm{~mm} \times 256 \mathrm{~mm}$ covering the motor cortex. 144 volumes ( 6 cycles) of fMRI were collected for each individual subject. In the second dataset, DTI was acquired from 10 healthy volunteers. The parameters of DTI are the same as the first dataset. The preprocessing steps are referred to [6].

\subsection{Gyri Segmentation}

We performed semi-automatic segmentation of two gyri, including precentral gyrus and postcentral gyrus in each hemisphere on the above reconstructed cortical surfaces from DTI. The gyral crest line (Fig. 2b) is defined as the shortest geometric path weighted by a value that is inversely proportional to the maximum principal curvature of surface (Fig. 2a) between two gyral end points on the surface. After the crest line has been selected, we resampled the crest line with 100 nodes with constant distance (Fig. 2c). After resampling, each crest line is composed by a sequence of 100 gyral nodes. After this, a rough correspondence is established between gyri across subjects by their node index, as illustrated in Fig. 2d.

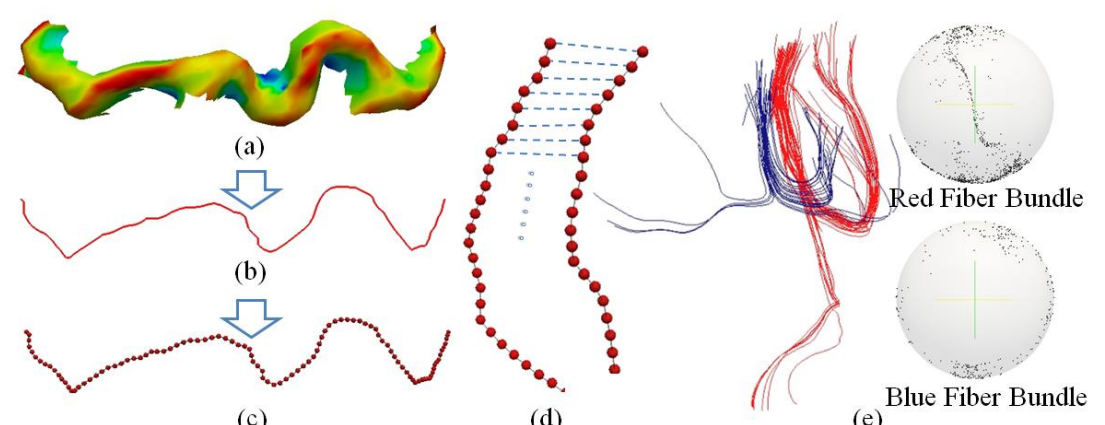

(c)

(d)

(e)

Fig. 2. Illustration of gyral crest line and fiber shape feature. (a) Surface's principal curvature. (b) Gyral crest line. (c) Resampled gyral crest line. (d) Correspondence between gyral nodes on the crest line. (e) Example of fiber bundle shape feature.

\subsection{Fiber Shape Feature}

We implemented a fiber shape descriptor that is akin to the recently published tracemap model in [7]. Briefly, a fiber tract was divided into overlapping segments. 
The principal direction of each segment was calculated using PCA and was represented by a point on a unit-sphere located at origin, as shown in Fig. 2e. The trace maps for each fiber of an ROI are accumulated and a density map was calculated and formulated as a 144-dimensional feature by discretizing the unit sphere and rearranging the values. Thus, each gyral node is represented by a 144-dimensional trace-map feature, which is quite descriptive in modeling different fiber shape patterns as shown in Fig. 2e. Notably, it has been widely reported that consistent structural connection patterns are predictive of brain function based on the general principle of "connectional fingerprint" introduced in [8], and thus it is expected that the used fiber shape descriptor is predictive of brain function, which will be verified by fMRI data later.

\subsection{Adaptive Multi-view Spectral Clustering (AMVSC)}

Spectral clustering is a technique that utilizes the properties of the Laplacian of the graph whose edges denote the similarity between the data points. The top $\mathrm{c}$ eigenvectors of the symmetric normalized graph Laplacian are the relaxations of the indicator matrix $\mathrm{G}$ which assigns each data point in the graph to one of the c clusters. To naturally integrate variable multi-view features of gyral nodes' shape patterns, we propose a unified objective function to simultaneously optimize clustering results of each individual view and their combinations. In other words, we minimize both the summation of the weighted spectral clustering error of each view and the distances between the multi-view clustering indicator matrix and the clustering indicator matrix of each single view. Thus, our multi-view spectral clustering objective function is the following:

$$
\begin{aligned}
& \min _{G, G^{k}, \alpha^{k}} \sum_{k=1}^{v}\left(\alpha^{k}\right)^{r} \operatorname{Tr}\left(G^{k T} L^{k} G^{k}\right)+\lambda \sum_{k=1}^{v} \operatorname{Tr}\left(\left(G-G^{k}\right)^{T}\left(G-G^{k}\right)\right) \\
& \text { s.t. } \quad G^{T} G=I, \quad \sum_{k=1}^{v} \alpha^{k}=1, \quad \alpha^{k} \geq 0
\end{aligned}
$$

where $\alpha^{k}$ is the normalized weight scalar for k-th view, which can be inferenced from the data directly. $L^{k} \in R^{n \times n}$ and $G^{k} \in R^{n \times c}$, are the corresponding Laplacian matrix and clustering indicator matrix of each view respectively $\forall k=1,2, \ldots, v . G \in R^{n \times c}$ is the multi-view clustering indicator matrix that we are interested in. $\lambda$ is the regularization parameter which controls the tradeoff between the clustering error and consistency and $r$ is the parameter that we use to control the distribution of the weight for each view. Specifically, when $r \rightarrow \infty$, we set up equal weight for each view; when $r \rightarrow 1$, only one weight is non-zero. Therefore, given the Laplacian matrix of each single view, we utilize Eq. (1) to learn the clustering indicator matrix of each view, clustering indicator matrix for multi-view and weight for each view simultaneously. 


\subsection{Optimization Algorithms}

We will resort to the following approach to solve Eq. (1) alternatively and iteratively. The first step is fixing $G, G^{K}$, solving $\alpha^{k}$.Then we need to solve the next problem,

$$
\min _{\alpha^{k}, \sum_{k=1}^{v} \alpha^{k}=1, \alpha^{k} \geq 0} \sum_{k=1}^{v}\left(\alpha^{k}\right)^{r} p^{k}
$$

Where $p^{k}=\operatorname{Tr}\left(G^{k^{T}} L^{k} G^{k}\right)$, and its and its Laplacian function is as follows,

$$
\sum_{k}^{v}\left(\alpha^{k}\right)^{r} p^{k}-\beta\left(\sum_{k=1}^{v} \alpha^{k}-1\right)
$$

where $\beta$ is the Laplacian multiplier. Setting the derivative of the Eq. (3) with respective $\alpha^{k}$ to zero, we have $\alpha^{k}=\left(\frac{\beta}{r p^{k}}\right)^{\frac{1}{r-1}}$ Substitute it into the constraint $\sum_{k=1}^{v} \alpha^{k}=1$, we obtain $\alpha^{k}=\left(r p^{k}\right)^{\frac{1}{1-r}} / \sum_{k=1}^{v}\left(r p^{k}\right)^{\frac{1}{1-r}}$.

The second step is fixing $\alpha^{k}$, solving $G$ and $G^{k}$. Let $\tilde{L}=\left(\alpha^{k}\right)^{r} L^{k}$, then the objective function becomes,

$$
\min _{G, G^{K}, G^{T}} \sum_{G=I}^{v} \operatorname{Tr}\left(G^{k^{T}} \tilde{L}^{k} G^{k}\right)+\lambda \sum_{k=1}^{v} \operatorname{Tr}\left(\left(G-G^{k}\right)^{T}\left(G-G^{k}\right)\right)
$$

And its Laplacian function is

$$
\min _{G, G^{k}} \sum_{k=1}^{v} \operatorname{Tr}\left(G^{k^{T}} \tilde{L}^{k} G^{k}\right)+\lambda \sum_{k=1}^{v} \operatorname{Tr}\left(\left(G-G^{k}\right)^{T}\left(G-G^{k}\right)\right)+\operatorname{Tr}\left(\Lambda^{T}\left(G^{T} G-I\right)\right)
$$

Where $\Lambda$ is the Laplacian multiplier. Setting the derivative of Eq. (4) with respective to $G^{k}$ to zero, then we get: $G^{k}=\lambda\left(\tilde{L}^{k}+\lambda I\right)^{-1} G$, where $I \in R^{n \times n}$ is the identity matrix. Substitute it into Eq. (4), then obtain the following equivalent problem,

$$
\left.\max _{G, G^{T} G=I} \operatorname{Tr}\left(G^{T}\left(\sum_{k=1}^{v} \tilde{L}^{k}+\lambda I\right)^{-1}\right) G\right)
$$

which can be tackled by traditional spectral clustering algorithm [9]. Repeating the above two steps alternatively, we iteratively update $\alpha^{k}$ (thus achieving adaptive MVSC) and $G$ until the objective function converges. We summarize the whole algorithm in Algorithm 1 [10]. 


\begin{tabular}{l}
\hline Algorithm 1. The algorithm of AMVSC \\
\hline Input:
\end{tabular}

1. The affinity matrix for each view $W^{k} \in R^{n \times n}, \forall k=1,2, \ldots, v$.

2. The number of clusters $c$, the regularization parameter $\lambda$ and the parameter $r$.

\section{Output:}

1.The cluster indication matrix $G \in R^{n \times c}$.

2.The weight for each view $\alpha^{k}$.

\section{Initialization:}

1.Set $\mathrm{t}=0$, and initialize the weight for each view, $\alpha_{t}^{1}=\alpha_{t}^{2}=\ldots=\alpha_{t}^{v}=\frac{1}{v}$.

\section{Procedure:}

1. Calculate the symmetric normalized graph Laplacian for each view $L^{k}=D^{k-1 / 2} W^{k} D^{k-1 / 2}$, where $D^{k}$ is the diagonal matrix with $d_{i i}^{k}=\sum_{j} w_{i j}^{k}$.

\section{Repeat}

1. Calculate $\tilde{L}_{t}^{k}=\left(\alpha_{t}^{k}\right)^{r} L^{k}$ and calculate $H_{t}=\sum_{k=1}^{v}\left(\tilde{L}_{t}^{k}+\lambda I\right)^{-1}$

2. Update $G_{t+1}$ by solving $\max _{G_{t}, G_{t}^{T} G_{t}=I} \operatorname{Tr}\left(G_{t}^{T} H_{t} G_{t}\right)$ by spectral clustering algorithm [9].

3. Update $\alpha_{t+1}^{k}$ by $\alpha_{t+1}^{k}=\left(r p_{t+1}^{k}\right)^{\frac{1}{1-r}} / \sum_{k=1}^{v}\left(r p_{t+1}^{k}\right)^{\frac{1}{1-r}}$, where $p_{t+1}^{k}=\operatorname{Tr}\left(G_{t+1}^{k}{ }^{T} L^{k} G_{t+1}^{k}\right)$

4. $t=t+1$

Until Converges

\section{Experimental Results}

\subsection{Gyral Parcellation Results}

Fig. 4 shows examples of parcellation results for the left pre-central gyrus and right post-central gyrus for 6 subjects, respectively. We set number of segments 10 which gives reasonable parcellation size (neither too small to obtain over segment, nor too large to cover multiple functional regions). By visual examination, the parcellated segments exhibit quite reasonably consistent patterns across different brains. In particular, the results in Figs. $3 a$ and $3 b$ and the results in Figs. $3 c$ and $3 d$ are from two separate datasets, suggesting that the proposed multi-view spectral clustering method really achieved reproducible and consistent parcellation results. To further verify our parcellation result, Figs. $4 \mathrm{a}$ and $4 \mathrm{~b}$ show the fiber shape patterns for all of the gyral segments and Fig. 4c shows the fiber shape patterns of the same segment in 10 brains. It is evident that the fiber shape patterns across different gyral segments are quite different, but the fiber shape patterns of the same gyral segment are quite consistent across individual brains. Quantitatively, the fiber shape similarities within the same corresponding gyral segment are much higher than those between different gyral segments, as shown in Fig. 5. On average, the within-segment similarity is 0.7 , while the between-segment similarity is 0.42 . 


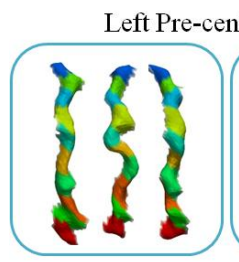

(a)

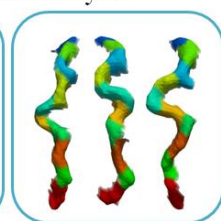

(b)

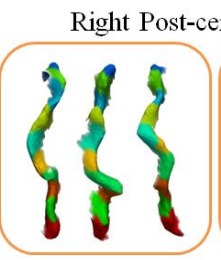

(c)

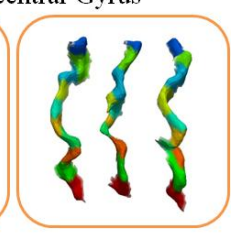

(d)

Fig. 3. The parcellation results of the left pre-central gyrus and right post-central gyrus of two datasets. (a) Left pre-central gyrus of subjects in the first dataset. (b) Left pre-central gyrus in the second dataset. (c) Right post-central gyrus in the first dataset. (d) Right post-central gyrus in the second dataset.

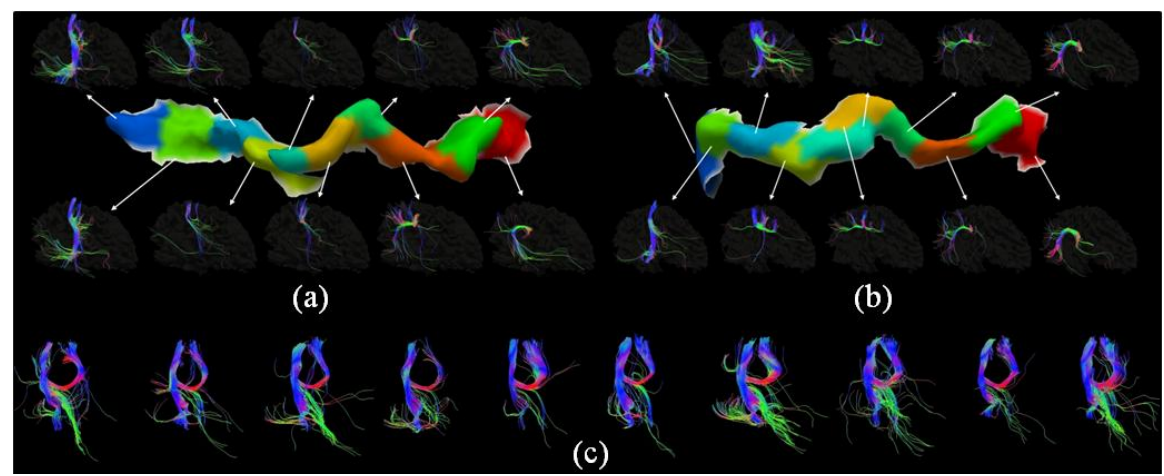

Fig. 4. Examples of fiber shape patterns of each cluster. (a) Fiber shape patterns of the left postcentral gyrus of one subject from the first dataset. (b) Fiber shape patterns of the left postcentral gyrus of one subject from the second dataset. (c) Examples of fiber shape patterns of the left most segment in (a).

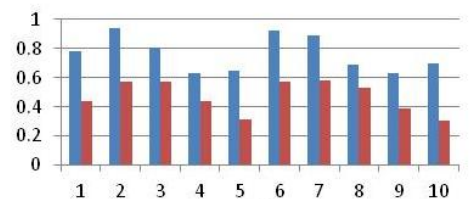

(a)

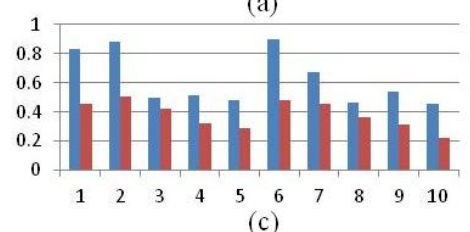

(c)

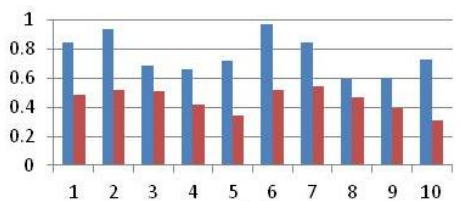

(b)

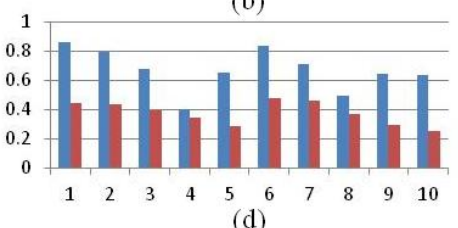

(d)

Fig. 5. Average similarity between fiber shape patterns within the same gyral segment in the second dataset. Blue bars: the similarities between subjects of the same segment. Red bars: the similarities between fiber shape patterns of different segments. The horizontal axis is gyral segment ID. (a) Left pre-central gyrus. (b) Right pre-central gyrus. (c) Left post-central gyrus. (d) Right post-central gyrus. 


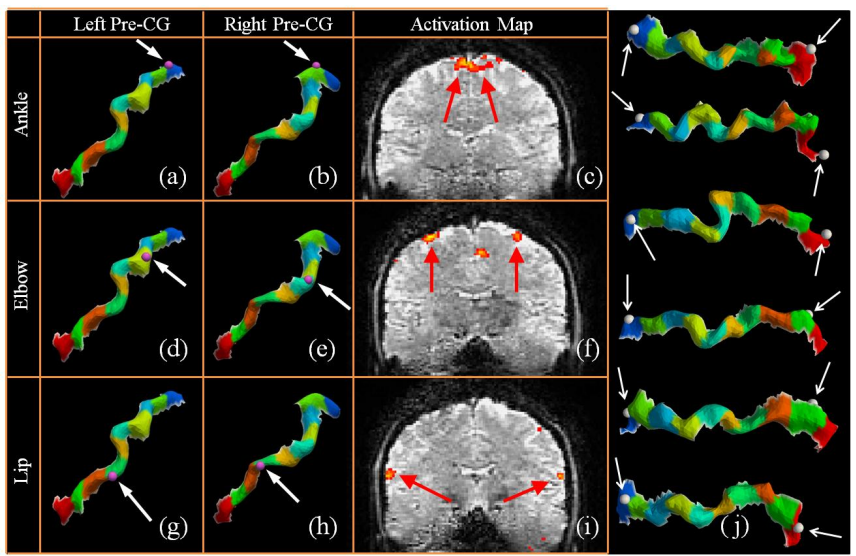

Fig. 6. Evaluation and validation of the pre-central gyrus parcellation by motor task-based fMRI [6] and consistent structural cortical landmarks [11]. (a)-(c) Ankle motor activation. (d)(f) Elbow motor activation. (g)-(i) Lip motor activation map. (j) Validation by consistent structure cortical landmarks.

\subsection{Validation via Motor Task-Based fMRI and Cortical Landmarks}

We used benchmark motor task-based fMRI data to validate the functional meanings and correspondences of those structurally parcellated gyral segments. As shown in Figs. 6a-6i, the fMRI-derived activations in responses to ankle, elbow and lip motor tasks are located on the same corresponding segments of the left and right precentral gyri. This result demonstrated that the structurally parcellated gyral segments possess functional correspondences and meanings. In a separate validation study shown in Fig. 6j, two cortical landmarks (highlighted by white arrows) identified in our recent data-drive discovery procedure [11] are located on two corresponding gyral segments across different brains as well, further suggesting that the clustered segments have functional meanings and possess correspondences across individuals.

\section{Discussion and Conclusion}

This paper presents a novel framework for group-wise consistent parcellation of cortical gyri via multi-view spectral clustering of DTI-derived fiber shape patterns. In this framework, each gyrus's nodes and their associated fiber shape patterns are considered as a separate view in the context of multi-view clustering framework, and gyral parcellation and their group-wise correspondences are achieved simultaneously via the novel multi-view spectral clustering algorithm. Our experimental results have shown that the precentral and postcentral gyri can be clustered into group-wise consistent and meaningful segments. Parts of the clustered results have been validated via benchmark cortical landmarks and task-based fMRI data. In the future, we plan to apply the similar methodology on other cortical gyri and validate the parcellation results via larger scale fMRI studies. The availability of fine-granularity parcellation of the cortex will enable many basic and clinical neuroscience applications [1]. 


\section{References}

1. Fischl, B., Sereno, M.I., Dale, A.M.: Cortical surface-based analysis II: inflation, flattening, and a surface-based coordinate system. Neuro Image 9, 195-207 (1999)

2. Shen, D., Davatzikos, C.: HAMMER: Hierarchical Attribute Matching Mechanism for Elastic Registration. IEEE Trans. on Medical Imaging 21(11), 1421-1439 (2002)

3. Li, G., Guo, L., Nie, J., Liu, T.: Automatic Cortical Sulcal Parcellation Based on Surface Principal Direction Flow Field Tracking. Neuroimage 46(4), 923-937 (2009)

4. Meier, J.D., Aflalo, T.N., Kastner, S., Graziano, M.S.: Complex Organization of Human Primary Motor Cortex: A High-Resolution fMRI Study. J. Neurophysiol. 100, 1800-1812 (2008)

5. Li, K., Guo, L., Li, G., Nie, J., Faraco, C., Zhao, Q., Miller, L., Liu, T.: Cortical surface based identification of brain networks using high spatial resolution resting state fMRI data. In: International Symposium of Biomedical Imaging (ISBI), pp. 656-659, 14-17 (2010)

6. Zhu, D., Li, K., Faraco, C., Deng, F., Zhang, D., Jiang, X., Chen, H., Guo, L., Miller, S., Liu, T.: Fine Granularity Parcellation of Gyrus via Fiber Shape and Connectivity Based Features. In: International Symposium of Biomedical Imaging (ISBI), pp. 817-821 (2011)

7. Zhu, D., Li, K., Faraco, C., Deng, F., Zhang, D., Jiang, X., Chen, H., Guo, L., Miller, S., Liu, T.: Optimization of Functional Brain ROIs via Maximization of Consistency of Structural Connectivity Profiles. NeuroImage 59(2), 1382-1393 (2011)

8. Passingham, R.E., Stephan, K.E., Kötter, R.: The anatomical basis of functional localization in the cortex. Nat. Rev. Neurosci. 3(8), 606-616 (2002)

9. Von Luxburg, U.: A tutorial on spectral clustering. Statistics and Computing 17(4), 395416 (2007)

10. Cai, X., Nie, F., Huang, H., Kamangar, F.: Heterogeneous Image Features Integration via Multi-Modal Spectral Clustering. In: CVPR, pp. 1977-1984 (2011)

11. Zhu, D., Li, K., Guo, L., Jiang, X., Zhang, T., Zhang, D., Chen, H., Deng, F., Faraco, C., Jin, C., Wee, C., Yuan, Y., Lv, P., Yin, Y., Hu, X., Duan, L., Hu, X., Han, J., Wang, L., Shen, D., Miller, L., Li, L., Liu, T.: DICCCOL: Dense Individualized and Common Connectivity-based Cortical Landmarks. Cerebral Cortex (in press, 2012) 